\title{
Interpreting the M2-brane Action
}

\author{
Shamik Banerjee and Ashoke Sen \\ Harish-Chandra Research Institute \\ Chhatnag Road, Jhusi, Allahabad 211019, INDIA \\ E-mail: bshamik@mri.ernet.in, sen@mri.ernet.in, ashokesen1999@gmail.com
}

\begin{abstract}
The world-volume theory of multiple M2-branes proposed recently has a free scalar field. For large vev of this scalar field the world-volume action reduces to that of multiple D2-branes with Yang-Mills coupling proportional to the vev. We suggest that the correct interpretation of this scalar field is as the radial position of the M2-brane center of mass in a cylindrical polar coordinate system. Regarding the azimuthal angle as compact we can regard this as a set of coincident D2-branes in type IIA string theory with varying dilaton and metric. We find that the effective world-volume theory on the D2-branes has Yang-Mills coupling proportional to the radial coordinate; furthermore the radial coordinate satisfies free field equations of motion. This agrees with the corresponding results derived from the M2-brane world-volume theory.
\end{abstract}


Since the discovery of a general class of $(2+1)$ dimensional superconformal field theories due to Bagger, Lambert and Gustavson [1, 2, 3, 4, 5], following earlier work of [6, 7], there has been much activity in trying to interpret these theories as the world-volume action of multiple M2-branes, as well as exploring various other aspects of these theories [8]- 36]. The simplest theory based on an $S O(4)$ algebra turned out to describe a theory of M2-branes on an orbifold [13, 14]. Furthermore, this theory has a novel Higgs mechanism whereby by giving vev to a scalar field on the world-volume we can recover the D2-brane action with an effective Yang-Mills coupling proportional to the vev [8]. While this result looked puzzling at the first sight, a simple interpretation was found in [14, where it was shown that in appropriate region of the M2-brane moduli space the transverse space of the M2-branes has a compact direction whose radius varies slowly as a function of one of the non-compact coordinates. By exploiting the duality between M-theory on a circle and type IIA string theory [37, 38], we can locally regard this theory as a type IIA string theory with slowly varying background and the M2branes as D2-branes moving in this background. This provides a natural explanation of how the vev of a world-volume scalar field could appear as a coupling constant of the D2-brane world-volume theory.

More recently refs. [25, 26, 27] proposed a theory of multiple M2-branes in flat space-time. The theory uses an indefinite metric in order to evade a no-go theorem [20, 22], and much is yet to be understood about this theory. However one of the puzzling features of this theory is that it has a free scalar field, and giving a large vev to this scalar again produces the worldvolume theory of multiple D2-branes whose Yang-Mills coupling is proportional to the scalar vev. Our goal in this note is to try to give an interpretation of this phenomenon along the lines of [14]. However our analysis will have much less control; unlike in the analysis of [14] where one could take an appropriate limit involving the order of the orbifold group and the scalar vev to keep the effective Yang-Mills coupling on the D2-branes small, we do not have such a control parameter. This however is to be expected if we are to describe M2-branes in flat eleven dimensional space-time where we have no adjustable coupling constant.

In a nutshell our proposal is the following. We split the eight transverse coordinates of the M2-brane into two sets, - two described using polar coordinate system and the rest of the six described using the Cartesian coordinates. Away from the origin of the polar coordinates, we can regard the azimuthal angle as a compact coordinate and regard the M2-branes as D2branes moving in a type IIA background whose dilaton and the metric vary as a function of the radial coordinate. A naive classical analysis shows that the effective Yang-Mills coupling 
constant on the D2-branes is proportional to the radial coordinate, thereby suggesting that we interpret the radial coordinate as the scalar field in the M2-brane world-volume theory whose vev controls the D2-brane coupling. Furthermore we can compute the equation of motion of the radial coordinate by regarding it as a scalar field on the D2-brane world-volume and find that it satisfies free field equations of motion. This agrees with the corresponding result derived from the M2-brane world-volume action.

We shall now proceed to describe our analysis. Let $x^{\mu}(0 \leq \mu \leq 2)$ be the world-volume coordinates of the M2-brane, $(r, \theta)$ denote the polar coordinates of two of the transverse directions of the M2-brane and $y^{m}(3 \leq m \leq 8)$ be the rest of the transverse directions. In this coordinate system the eleven dimensional flat metric is given by

$$
d s_{11}^{2}=\eta_{\mu \nu} d x^{\mu} d x^{\nu}+\left(d r^{2}+r^{2} d \theta^{2}\right)+d y^{m} d y^{m} .
$$

We shall now interprete the coordinate $\theta$ as the compact direction and regard the theory as type IIA string theory in a space-time labelled by $(\vec{x}, r, \vec{y})$. Then by standard rules [38] the type IIA dilaton $\phi$ and the type IIA metric $d s_{I I A}^{2}$ are given by

$$
e^{2 \phi}=r^{3}, \quad d s_{I I A}^{2} \equiv\left(g_{\mu \nu} d x^{\mu} d x^{\nu}+G_{r r} d r^{2}+G_{m n} d y^{m} d y^{n}\right)=r\left(\eta_{\mu \nu} d x^{\mu} d x^{\nu}+d r^{2}+d y^{m} d y^{m}\right)
$$

In this picture $N$ coincident M2-branes placed at $(r, \theta, \vec{y})$ can be regarded as $N$ coincident D2branes placed at $(r, \vec{y})$. The part of the D2-brane world-volume action containing the $S U(N)$ gauge fields will be given by

$$
-\frac{1}{4} \int d^{3} x e^{-\phi} \sqrt{-\operatorname{det} g} g^{\mu \rho} g^{\nu \sigma} \operatorname{Tr}\left[F_{\mu \nu} F_{\rho \sigma}\right]=-\frac{1}{4} \int d^{3} x r^{-2} \eta^{\mu \rho} \eta^{\nu \sigma} \operatorname{Tr}\left[F_{\mu \nu} F_{\rho \sigma}\right] .
$$

From this we see that the effective Yang-Mills coupling $g_{Y M}$ is given by

$$
g_{Y M}=r .
$$

On the other hand the world-volume scalar field $R$ on the M2-brane, labelling the centre of mass $r$ coordinate, has an action proportional to

$$
-\frac{1}{2} \int d^{3} x\left[e^{-\phi} \sqrt{-\operatorname{det} g} g^{\mu \nu} G_{r r}\right]_{r=R} \partial_{\mu} R \partial_{\nu} R=-\frac{1}{2} \int d^{3} x \eta^{\mu \nu} \partial_{\mu} R \partial_{\nu} R
$$

Thus the world-volume field $R$ satisfies the free field equation of motion

$$
\eta^{\mu \nu} \partial_{\mu} \partial_{\nu} R=0
$$


and the vev of this field determines the $r$-coordinate of the branes.

Let us compare this with the results of [25, 26, 27]. For definiteness we shall use the convention of [25]. In this paper the membrane action contains a scalar field $X^{-(8)}$ whose vacuum expectation value controls the strength of the Yang-Mills coupling. Thus we can identify it with the scalar field $R$ introduced above. Furthermore the field $X^{-(8)}$ is known to satisfy the equations of motion of a free field:

$$
\eta^{\mu \nu} \partial_{\mu} \partial_{\nu} X^{-(8)}=0
$$

This agrees with (6), providing a non-trivial consistency check on our proposal 1

If this proposal is correct then this implies that the apparent dependence of the M2-brane world-volume theory on the vacuum expectation value of the field $X^{-(8)}$ is fake; different vacuum expectation values of $X^{-(8)}$ correspond to placing the membrane at different values of the radial coordinate $r$. It will be interesting to see if this symmetry is present in the theories proposed in [25, 26]; this will involve a non-trivial transformation on the fields since from the geometric viewpoint described above this will amount to transforming from polar coordinates around a given origin to the polar coordinates around a different origin. It is of course possible that the symmetry is not visible in the classical theory, and only after quantization we shall discover this symmetry. This also suggests that there may be a simpler version of the M2brane world-volume theory that uses the Cartesian coordinate system instead of cylindrical polar cordinates for describing the transverse space.

\section{References}

[1] J. Bagger and N. Lambert, "Modeling multiple M2's," Phys. Rev. D 75, 045020 (2007) arXiv:hep-th/0611108.

[2] A. Gustavsson, "Algebraic structures on parallel M2-branes," arXiv:0709.1260 [hep-th].

[3] J. Bagger and N. Lambert, "Gauge Symmetry and Supersymmetry of Multiple M2-Branes," Phys. Rev. D 77, 065008 (2008) arXiv:0711.0955 [hep-th]].

[4] J. Bagger and N. Lambert, "Comments On Multiple M2-branes," JHEP 0802, 105 (2008) arXiv:0712.3738 [hep-th]].

[5] A. Gustavsson, "Selfdual strings and loop space Nahm equations," JHEP 0804, 083 (2008) arXiv:0802.3456 [hep-th]].

\footnotetext{
${ }^{1}$ Note that for large $r$, 1.e. large $X^{-(8)}$ where the M2-brane theory reduces to the D2-brane theory, the string coupling is large but the curvature and the dilaton gradient measured in the string scale is small.
} 
[6] J. H. Schwarz, "Superconformal Chern-Simons theories," JHEP 0411, 078 (2004) arXiv:hep-th/0411077.

[7] A. Basu and J. A. Harvey, "The M2-M5 brane system and a generalized Nahm's equation," Nucl. Phys. B 713, 136 (2005) arXiv:hep-th/0412310].

[8] S. Mukhi and C. Papageorgakis, "M2 to D2," arXiv:0803.3218 [hep-th].

[9] M. A. Bandres, A. E. Lipstein and J. H. Schwarz, "N = 8 Superconformal Chern-Simons Theories," JHEP 0805, 025 (2008) arXiv:0803.3242 [hep-th]].

[10] D. S. Berman, L. C. Tadrowski and D. C. Thompson, "Aspects of Multiple Membranes," arXiv:0803.3611 [hep-th].

[11] M. Van Raamsdonk, "Comments on the Bagger-Lambert theory and multiple M2-branes," arXiv:0803.3803 [hep-th].

[12] A. Morozov, "On the Problem of Multiple M2 Branes," arXiv:0804.0913 [hep-th].

[13] N. Lambert and D. Tong, "Membranes on an Orbifold," arXiv:0804.1114 [hep-th].

[14] J. Distler, S. Mukhi, C. Papageorgakis and M. Van Raamsdonk, "M2-branes on M-folds," JHEP 0805, 038 (2008) arXiv:0804.1256 [hep-th]].

[15] U. Gran, B. E. W. Nilsson and C. Petersson, "On relating multiple M2 and D2-branes," arXiv:0804.1784 [hep-th].

[16] P. M. Ho, R. C. Hou and Y. Matsuo, "Lie 3-Algebra and Multiple M2-branes," arXiv:0804.2110 [hep-th].

[17] J. Gomis, A. J. Salim and F. Passerini, "Matrix Theory of Type IIB Plane Wave from Membranes," arXiv:0804.2186 [hep-th].

[18] E. A. Bergshoeff, M. de Roo and O. Hohm, "Multiple M2-branes and the Embedding Tensor," arXiv:0804.2201 [hep-th].

[19] K. Hosomichi, K. M. Lee and S. Lee, "Mass-Deformed Bagger-Lambert Theory and its BPS Objects," arXiv:0804.2519 [hep-th].

[20] G. Papadopoulos, "M2-branes, 3-Lie Algebras and Plucker relations," JHEP 0805, 054 (2008) arXiv:0804.2662 [hep-th]].

[21] D. Gaiotto and E. Witten, "Janus Configurations, Chern-Simons Couplings, And The ThetaAngle in N=4 Super Yang-Mills Theory," arXiv:0804.2907 [hep-th].

[22] J. P. Gauntlett and J. B. Gutowski, "Constraining Maximally Supersymmetric Membrane Actions," arXiv:0804.3078 [hep-th].

[23] G. Papadopoulos, "On the structure of k-Lie algebras," arXiv:0804.3567] [hep-th]. 
[24] P. M. Ho and Y. Matsuo, "M5 from M2," arXiv:0804.3629 [hep-th].

[25] J. Gomis, G. Milanesi and J. G. Russo, "Bagger-Lambert Theory for General Lie Algebras," arXiv:0805.1012 [hep-th].

[26] S. Benvenuti, D. Rodriguez-Gomez, E. Tonni and H. Verlinde, "N=8 superconformal gauge theories and M2 branes," arXiv:0805.1087 [hep-th].

[27] P. M. Ho, Y. Imamura and Y. Matsuo, "M2 to D2 revisited," arXiv:0805.1202 [hep-th].

[28] A. Morozov, "From Simplified BLG Action to the First-Quantized M-Theory," arXiv:0805.1703 [hep-th].

[29] Y. Honma, S. Iso, Y. Sumitomo and S. Zhang, "Janus field theories from multiple M2 branes," arXiv:0805.1895 [hep-th].

[30] H. Fuji, S. Terashima and M. Yamazaki, "A New N=4 Membrane Action via Orbifold," arXiv:0805.1997 [hep-th].

[31] P. M. Ho, Y. Imamura, Y. Matsuo and S. Shiba, "M5-brane in three-form flux and multiple M2-branes," arXiv:0805.2898 [hep-th].

[32] C. Krishnan and C. Maccaferri, "Membranes on Calibrations," arXiv:0805.3125 [hep-th].

[33] Y. Song, "Mass Deformation of the Multiple M2 Branes Theory," arXiv:0805.3193 [hep-th].

[34] I. Jeon, J. Kim, N. Kim, S. W. Kim and J. H. Park, "Classification of the BPS states in BaggerLambert Theory," arXiv:0805.3236 [hep-th].

[35] M. Li and T. Wang, "M2-branes Coupled to Antisymmetric Fluxes," arXiv:0805.3427 [hep-th].

[36] K. Hosomichi, K. M. Lee, S. Lee, S. Lee and J. Park, "N=4 Superconformal Chern-Simons Theories with Hyper and Twisted Hyper Multiplets," arXiv:0805.3662 [hep-th].

[37] P. K. Townsend, "The eleven-dimensional supermembrane revisited," Phys. Lett. B 350, 184 (1995) arXiv:hep-th/9501068.

[38] E. Witten, "String theory dynamics in various dimensions," Nucl. Phys. B 443, 85 (1995) arXiv:hep-th/9503124]. 